\title{
Spatially Mapping Heterogeneous Nucleation Kinetics of Silver Nanocrystals with Liquid Cell Scanning Transmission Electron Microscopy
}

\author{
M. Wang, ${ }^{1}$ T.U. Dissanayake, ${ }^{1}$ C. Park, ${ }^{2}$ T.J. Woehl ${ }^{1, *}$ \\ ${ }^{1}$ Department of Chemical and Biomolecular Engineering, University of Maryland, College Park, College \\ Park, MD USA \\ ${ }^{2}$ Department of Industrial and Manufacturing Engineering, Florida State University, Tallahassee, FL USA \\ *Corresponding author: tjwoehl@umd.edu
}

Nucleation is ubiquitous during liquid phase crystallization processes, both in natural and synthetic systems [1], and controls phenomena such as polymorph selection during crystallization of drug molecules and proteins, biomineral nucleation, in vivo aggregation of pathogenic proteins, and synthesis of nanomaterials. Except for very clean systems, nucleation is almost always heterogeneous because the presence of a solid interface significantly decreases the free energy barrier to nucleation [2]. The free energy barrier for a given heterogeneous nucleation site depends sensitively on the local surface topography and surface chemistry (e.g. functional groups). The identity and kinetics of nucleation at heterogeneous nucleation sites on a liquid-solid interface remains elusive because it is challenging to determine the free energy barrier for a given nucleation site with high spatial resolution.

Liquid cell scanning transmission electron microscopy (LC-STEM) and electron beam induced nanocrystal growth provide a unique approach for visualizing heterogenous nucleation at high spatial and temporal resolution [3, 4]. In this method, the electron beam stimulates nucleation and growth of nanocrystals from a precursor solution while simultaneously visualizing the process in real time [5]. Nanocrystals form at the liquid-solid interface between the precursor solution and the silicon nitride ( $\mathrm{SiN})$ membranes. Here we demonstrate spatial mapping of heterogeneous nucleation kinetics occurring during electron beam induced silver nanocrystal formation with LC-STEM. Nanocrystal formation from a silver nitrate solution is stimulated by the STEM electron beam under kinetically controlled conditions, including control over precursor concentration and reducing agent concentration [6]. We performed single particle kinetic measurements of the nucleation induction time $\left(t_{\text {ind }}\right)$ and the growth rate for each of $\sim 500$ nanocrystals in a single experiment.

Silver nanocrystals were grown using electron beam induced growth and in situ movies formed by continuously imaging the samples in BF-STEM mode (Figure 1). Image analysis was used to extract the nucleation induction time of each nanocrystal, which is defined as the time elapsed between the beginning of an experiment and the detection of a nanocrystal with image analysis; it is inversely proportional to the nucleation rate (Figure 2a). The nucleation induction times for each nanocrystal were spatially correlated with the position of the nanocrystal in the BF-STEM image and a heat map of the spatially varying nucleation kinetics was generated by interpolation (Figure 2b). Strikingly, the heat maps revealed spatial variations in the local nucleation kinetics on the $\mathrm{SiN}$ membrane over spatial scales as small as $\sim 10 \mathrm{~nm}$. The heat map in Figure 2b shows $\sim 50-200 \mathrm{~nm}$ sized islands (blue/teal) of rapidly nucleating nanocrystals with $t_{\text {ind }}<20$ seconds. These islands were surrounded by a continuous phase of slowly nucleating nanocrystals with $t_{\text {ind }}>30$ seconds. We interpret this phenomenon in terms of heterogeneous nucleation. Nucleation sites with low free energy barriers, such as areas of the silicon nitride membranes with high densities of negatively charged silanol functional groups, show locally rapid nucleation, while areas with higher free energy barriers showed slow nucleation. We will present chemical force microscopy images and a semi-quantitative phenomenological model derived from classical nucleation theory $(\mathrm{CNT})$ 
to further support our interpretation. Overall, we show that LC-STEM is a powerful tool for probing heterogeneous nucleation kinetics at nanometer scale spatial resolutions [7].

\section{References:}

[1] D. Erdemir, A.Y. Lee, A.S. Myerson, Accounts of Chemical Research, 42 (2009) 621-629.

[2] R.P. Sear, CrystEngComm, 16 (2014) 6506-6522.

[3] H.M. Zheng, R.K. Smith, Y.W. Jun, C. Kisielowski, U. Dahmen, A.P. Alivisatos, Science, 324 (2009) 1309-1312.

[4] T.J. Woehl, J.E. Evans, L. Arslan, W.D. Ristenpart, N.D. Browning, Acs Nano, 6 (2012) 8599-8610.

[5] H.G. Liao, K.Y. Niu, H.M. Zheng, Chemical Communications, 49 (2013) 11720-11727.

[6] M. Wang, C. Park, T.J. Woehl, Chemistry of Materials, 30 (2018) 7727-7736.

[7] We acknowledge funding from ORAU and University of Maryland Energy Research Center.
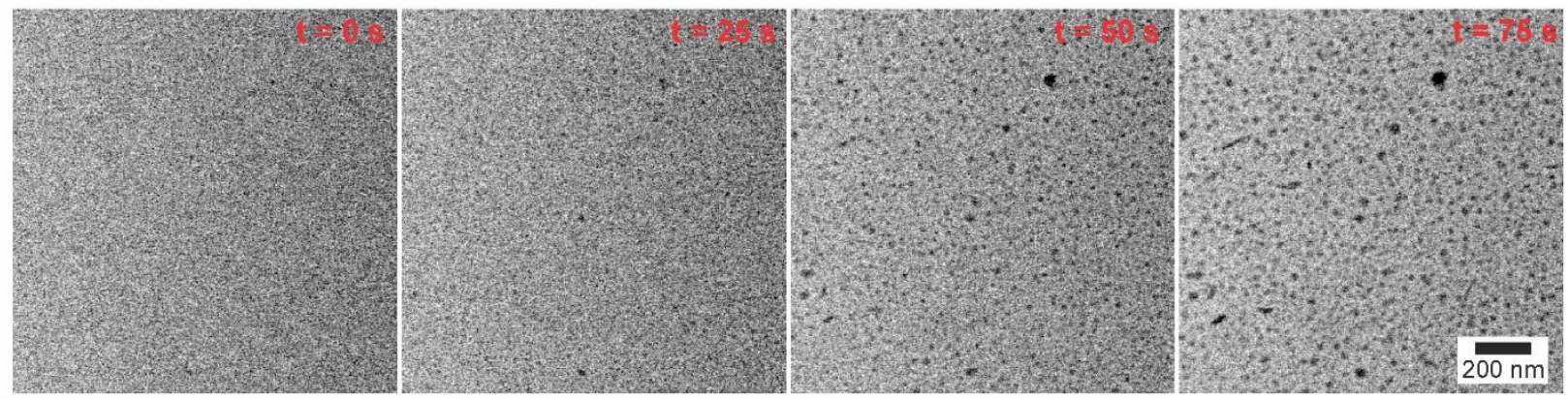

Figure 1. Time-lapsed series of BF-STEM images showing electron beam induced nucleation and growth of silver nanocrystals on a $\mathrm{SiN}$ membrane. The magnification was $\mathrm{M}=100,000 \mathrm{x}$ and the beam current was $21 \mathrm{pA}$, yielding a dose rate of $0.5 \mathrm{MGy} / \mathrm{s}$.

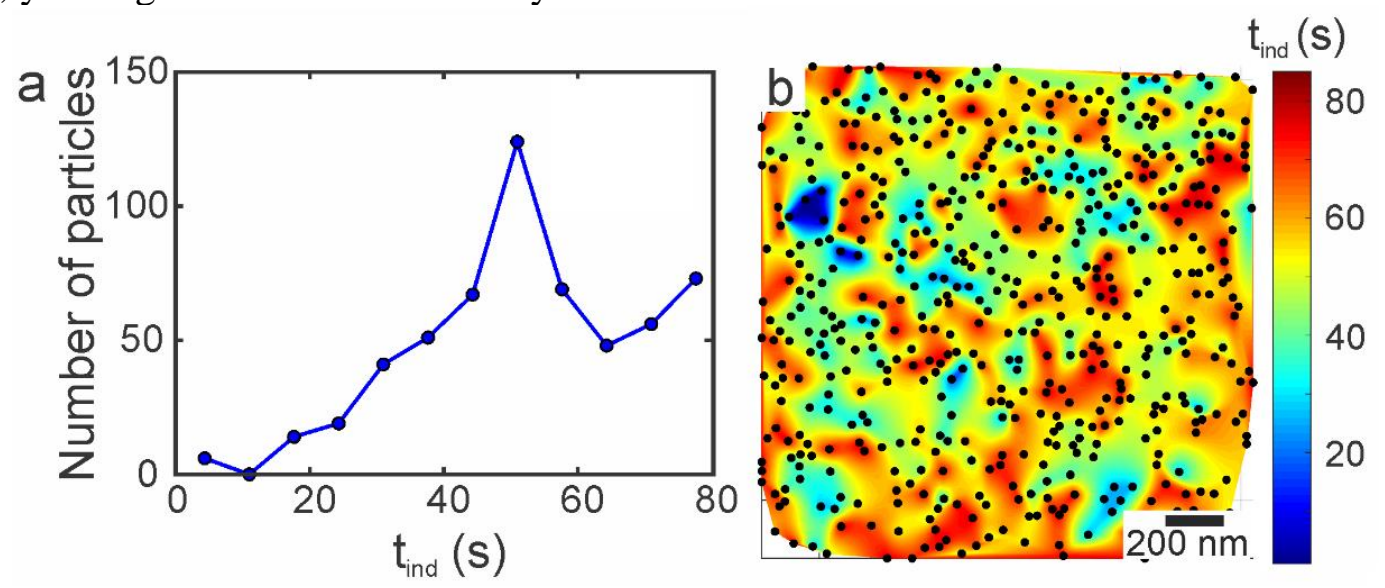

Figure 2. (a) Histogram of nucleation induction times for all silver nanocrystals shown in Figure 1. (b) Spatial heat map of the nucleation induction times for each nanocrystal. The black dots are the centroids of each nanocrystal, while the coloring represents the interpolated nucleation induction time. Red and orange colors represent locations slow nucleation kinetics while blue and teal represent fast nucleation. 